CD137 Pathway:

Immunology and Diseases 


\section{CD137 Pathway: Immunology and Diseases}

Edited by

LIEPING CHEN

John Hopkins University

Baltimore, MD, USA

睹 Springer 
Editor:

Lieping Chen

Department of Dermatology and Oncology

Johns Hopkins University School of Medicine

600 N. Wolf Street, Jefferson 1-121

Baltimore, MD 21205

Ichen42@jhmi.edu

Library of Congress Control Number: 2006933637

ISBN 10: 0-387-31322-2

ISBN 13: 978-0-387-31322-1

Printed on acid-free paper.

(C) 2006 Springer Science+Business Media, LLC.

All rights reserved. This work may not be translated or copied in whole or in part without the written permission of the publisher (Springer Science+Business Media, Inc., 233 Spring Street, New York, NY 10013, USA), except for brief excerpts in connection with reviews or scholarly analysis. Use in connection with any form of information storage and retrieval, electronic adaptation, computer software, or by similar or dissimilar methodology now known or hereafter developed is forbidden.

The use in this publication of trade names, trademarks, service marks, and similar terms, even if they are not identified as such, is not to be taken as an expression of opinion as to whether or not they are subject to proprietary rights.

\section{$\begin{array}{lllllllll}9 & 8 & 7 & 6 & 5 & 4 & 3 & 2 & 1\end{array}$}

springer.com 


\section{Preface}

It is still hard to believe that manipulation of a single protein on the cell surface or an interaction of two or more proteins, which at times is collectively referred to as a "pathway," could have such a profound effect on our immune system. At present, a number of such proteins or pathways have been identified. These observations could only be interpreted in a way that, although tens of thousand of proteins are required for a perfectly healthy immune system, many of these pathways may work in either interconnected or linear fashion. Therefore, the combined understanding of each pathway, their interactions with other pathways, and the functional consequence, is a cornerstone for our interpretation of pathological basis of diseases and future treatments. It is important to stay abreast on the pace of progress, which I refer to as periodic summary of incremental and breakthrough discoveries in each pathway by the experts and the leader in the field. The CD137 Pathway: Immunology and Diseases represents such an effort and this is the first attempt to summarize our understanding of the CD137 pathway. The following chapters cover the majority of active areas of research in this pathway and also provide an essential resource to both the nonexperts and experts in the field.

I would like to thank all of the authors for their contributions with this work and my administrative assistant, Jennifer Osborne, for her patience and assistance with editing. I would also like to thank The Johns Hopkins Medical Institutions and The National Institutes of Health for their financial support.

Lieping Chen 


\section{Contents}

1. Genes, Transcripts and Proteins of CD137 Receptor and Ligand

Dass S. Vinay and Byoung S. Kwon

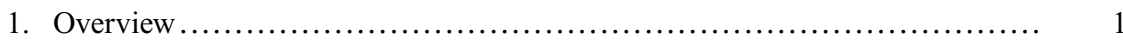

2. Discovery, Alternative Names, and Structure of CD137 ...................... 3

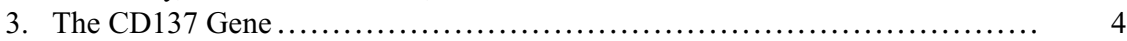

4. The CD137 Protein ....................................................... 5

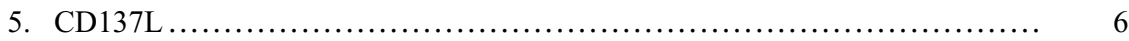

5.1. The Discovery of CD137L ........................................ 6

5.2. CD137L Structure and Expression.................................... 6

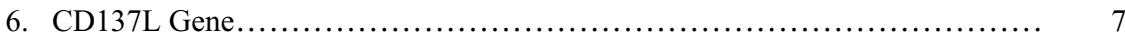

7. CD137L Protein ....................................................... 7

7.1. Regulation of RNA and Protein Expression ............................. 8

7.2. CD137-CD137L in Health and Disease................................... 9

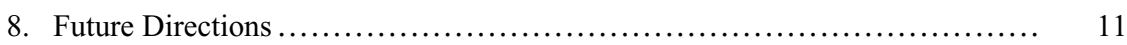

Acknowledgments ..................................................... 11

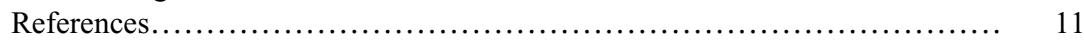

2. CD137 Signal Transduction 15

Hyeon-Woo Lee and Byoung S. Kwon

1. Background .............................................................. 15

2. CD137 (4-1BB) As an Immune Stimulator ..................................... 16

3. CD137 Signal Transduction ........................................... 17

4. Concluding Remarks .................................................. 22

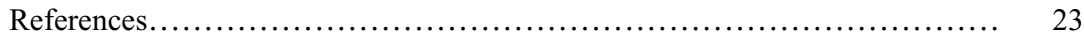

3. Significance of Reverse Signal Transduction for the Biology of the CD137 Receptor/Ligand System Herbert Schwarz

1. Biology of Reverse Signaling Through CD137 Ligand ....................... 30

1.1. CD137 Ligand Activities on Monocytes and Macrophages ............... $\quad 30$

1.2. CD137 Ligand Activities on Dendritic Cells ............................. 34

1.3. CD137 Ligand Activities on B Cells................................... 34

1.4. CD137 Ligand Activities on Bone Marrow Cells........................ 35

1.5. CD137 Ligand Activities on T Cells .................................. 36

1.6. CD137 Ligand Activities on Non-hematopoietic Cells................... 37 
1.7. CD137 Ligand Signal Transduction Pathway ........................ 39

1.8. Regulation of CD137 Ligand Signaling .............................. 39

1.9. Influence of Soluble CD137 and Soluble CD137 Ligand on CD137 Ligand Signaling

2. Bidirectional Signaling in Other Receptor/Ligand Systems ................. 40

3. Concluding Remarks ............................................. 41

References......................................................... 42

4. CD137 Signal in the Regulation of Innate Immunity Lieping Chen

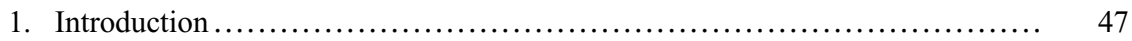

2. NK Cells ..................................................... 47

3. Macrophages/Monocyte.................................................. 49

4. Dendritic Cells ..................................................... 50

5. Granulocytes ........................................................ 51

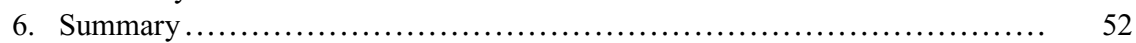

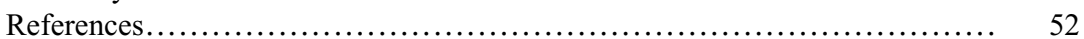

\section{Regulation of T Cell-Dependent Humoral Immunity} Through CD137 (4-1BB) Mediated Signals

Robert S. Mittler, Liguo Niu, Becker Hewes, and Juergen Foell

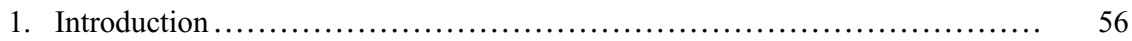

2. T and B Cell Activation and Costimulation............................. 56

3. CD137 Expression and T Cell Costimulation .............................. 59

4. Anti-CD137-Mediated Suppression of Humoral Immunity ................. 60

5. Anti-CD137 Induced Suppression of Autoantibodies....................... 64

6. DC Function, CD137 Expression and Signaling ........................... 69

7. Anti-tumor Immunity and B Cells.................................... 72

8. Anti-CD137 mAbs Disrupt Hematopoiesis in Mice. ........................ 74

9. Concluding Remarks ............................................. 77

References..................................................... 77

6. CD137 in the Regulation of T Cell Response to Antigen 83 Yuwen Zhu and Lieping Chen

1. CD137 in Naïve T Cell Costimulation ............................... 83

1.1. Effects of CD137 Engagement on CD8 ${ }^{+} \mathrm{T}$ Cells ..................... 83

1.2. Effects of CD137 on CD8 ${ }^{+} \mathrm{T}$ Cell Priming, Division and Survival ........ 85

1.3. Effects of CD137 on CD4 ${ }^{+}$T Cells: Positive or Negative?............... 86

2. CD137 and Regulatory T Cells (Treg) ................................ 87

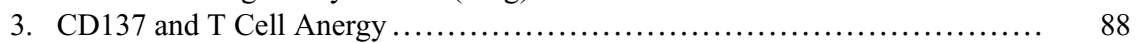

4. CD137 and Memory T Cell (Tm) Response............................. 90

5. Conclusions and Perspectives ........................................ 92

References...................................................... 92 
7. Autoimmune Diseases

Yonglian Sun and Yang-Xin Fu

1. Introduction

2. Role of CD137/CD137L Interaction in the Pathogenesis of Autoimmune Diseases.............................................. 98

2.1. Lack of CD137/CD137L Interaction Prevents Autoimmune Diseases ..... 98

2.2. Soluble CD137 and Autoimmune Diseases .......................... 99

3. Treatment of Autoimmune Disease with Agonistic Anti-CD137 ............. 100

3.1. Experimental Autoimmune Encephalomyelitis (EAE)................ 101

3.2. Experimental Autoimmune Uveitis (EAU) ........................ 102

3.3. Systemic Lupus Erythematosus (SLE)........................... 103

3.4. Collagen-induced Arthritis (CIA) ............................. 105

3.5. Chronic Graft-Versus-Host Disease (cGVHD) ..................... 105

4. Mechanisms Involved in CD137 Agonist-mediated Inhibition of Autoimmune Diseases........................................... 106

4.1. Apoptosis of T Lymphocytes ..................................... 106

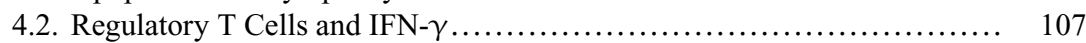

4.3. Helper T Cell Anergy .......................................... 108

4.4. B Cell Apoptosis .............................................. 109

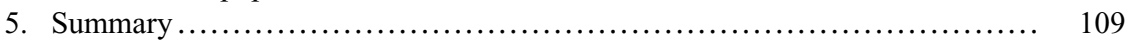

References....................................................... 110

\section{CD137/CD137 Ligand in Tumor and Viral Immunotherapy 117}

Ignacio Melero, Oihana Murillo, Iñigo Tirapu, Eduardo Huarte, Ainhoa Arina, Laura Arribillaga, and Juan José Lasarte

1. CD137 (4-1BB) and CD137 Ligand (4-1BB-Ligand) Meet Tumor Immunology.

1.1. The Arrival of Agonistic Anti-CD137 Monoclonal Antibodies........... 117

1.2. A Comparison with Anti-CTLA-4 Monoclonal Antibodies............... 118

1.3. Transfection of CD137 Ligand into Malignant Cells ................... 120

2. Developments and Improvements on CD137/CD137 Ligand Therapeutic Strategies

2.1. Immunization: CD137 Breaks Ignorance and Tolerance.

2.2. CD137 as an Adjuvant for Adoptive T Cell Therapy and Bone Marrow Transplantation.

2.3. CD137 Acting in Synergy with Cytokines and Other Costimulatory Molecules

3. CD137/CD137 Ligand in the Antiviral Immune Response and in Viral Vaccination .................................................. 126

4. Reflections on the Mechanism(s) of Action........................... 127

5. Using Preclinical Information for Clinical Development of Immunotherapy .... 128 References................................................... 130

Index 


\section{Contributors}

\section{Ainhoa Arina}

Centro de Investigación Médica

Aplicada y Clínica Universitaria

Universidad de Navarra

Pamplona, Spain

\section{Becker Hewes}

Department of Pediatric Hematology and Oncology

Emory University School of Medicine

954 Gatewood Road

Atlanta, GA 30329

\section{Byoung S. Kwon}

Department of Ophthalmology

LSU Eye Center, Louisiana State University

Health Sciences Center

New Orleans, LA

USA

\section{Dass S. Vinay}

Department of Ophthalmology

LSU Eye Center, Louisiana State University

Health Sciences Center

New Orleans, LA

USA

\section{Eduardo Huarte}

Centro de Investigación Médica

Aplicada y Clínica Universitaria

Universidad de Navarra

Pamplona, Spain

\section{Herbert Schwarz}

Department of Physiology,

National University of Singapore

2 Medical Drive, MD 9

Singapore 117597

\section{Hyeon-Woo Lee}

Department of Pharmacology

School of Dentistry

Kyung Hee University

Seoul 130-701, Korea

\section{Ignacio Melero}

Centro de Investigación Médica

Aplicada y Clínica Universitaria

Universidad de Navarra

Pamplona, Spain

\section{Iñigo Tirapu}

Centro de Investigación Médica

Aplicada y Clínica Universitaria

Universidad de Navarra

Pamplona, Spain

\section{Juan José Lasarte}

Centro de Investigación Médica

Aplicada y Clínica Universitaria

Universidad de Navarra

Pamplona, Spain

\section{Juergen Foell}

Division of Pediatrics, Hematology, Oncology, and Immunology

Martin Luther University

Halle-Wittenberg, 06097

Halle, Germany

\section{Laura Arribillaga}

Centro de Investigación Médica

Aplicada y Clínica Universitaria

Universidad de Navarra

Pamplona, Spain

\section{Lieping Chen}

Department of Dermatology and Oncology Johns Hopkins University School of Medicine 600 N. Wolf Street, Jefferson 1-121

Baltimore, MD 21205

\section{Liguo Niu}

Emory Vaccine Center, Emory University

School of Medicine

954 Gatewood Road

Atlanta, GA 30329

\section{Oihana Murillo}

Centro de Investigación Médica Aplicada y Clínica Universitaria

Universidad de Navarra

Pamplona, Spain 
Robert S. Mittler

Department of Surgery, Emory Vaccine Center

Emory University School of Medicine

954 Gatewood Road

Atlanta, GA 30329

\section{Yang-Xin Fu}

The Department of Pathology and Committee in Immunology

The University of Chicago

Chicago, Illinois, USA

\section{Yonglian Sun}

The Department of Pathology and Committee in Immunology

The University of Chicago

Chicago, Illinois, USA

\section{Yuwen Zhu}

Department of Dermatology and Oncology

Johns Hopkins University School of Medicine

Baltimore, MD

USA 